\title{
Momentum Flux Measuring Instrument for Neutral and Charged Particle Flows
}

Greg Chavers

Propulsion Research Center, Marshall Space Flight Center, NASA, Huntsville, Alabama 35812

Franklin Chang-Diaz

Advanced Space Propulsion Laboratory, Johnson Space Center, NASA, Houston, Texas 77059

\begin{abstract}
An instrument to measure the momentum flux (total pressure) of plasma and neutral particle jets onto a surface has been developed. While this instrument was developed for magnetized plasmas, the concept works for non-magnetized plasmas as well. We have measured

forces as small as $10^{-4}$ Newtons on a surface immersed in the plasma where small forces are due to ionic and neutral particles with kinetic energies on the order of a few eV impacting the surface. This instrument, a force sensor, uses a target plate (surface) that is immersed in the plasma and connected to one end of an alumina rod while the opposite end of the alumina rod is mechanically connected to a titanium beam on which four strain gauges are mounted. The force on the target generates torque causing strain in the beam. The resulting strain measurements can be correlated to a force on the target plate. The alumina rod electrically and thermally isolates the target plate from the strain gauge beam and allows the strain gauges to be located out of the plasma flow while also serving as a moment arm of several inches to increase the strain in the beam at the strain gauge location. These force measurements correspond directly to momentum flux and may be used with known plasma conditions to place boundaries on the kinetic energies of the plasma and neutral particles. The force measurements may also be used to infer thrust produced by a plasma propulsive device. Stainless steel, titanium, molybdenum, and aluminum
\end{abstract}


flat target plates have been used. Momentum flux measurements of $\mathrm{H} 2, \mathrm{D} 2, \mathrm{He}$, and Ar plasmas produced in a magnetized plasma device have been performed. 


\section{Introduction}

To better understand the characteristics of a combined flow of neutral and charged particles onto a solid surface, an instrument was developed to measure the momentum flux from neutral and plasma jets. Electrostatic probes may be used to determine the particle and energy fluxes locally in the plasma. However, complications caused by collision processes such as charge exchange limit the calculation accuracy of the momentum flux or pressure onto the solid surface using the particle and energy flux data. Direct measurement of the momentum flux affords the surface interactions such as particle and energy reflections from the surface to be evaluated. The momentum flux data, with heat flux data, may be used to deduce information about the plasma-surface interaction. The forces measured from these plasma / neutral jets range from $10^{-4}$ to $10^{-1}$ Newtons with ionic impact energies on the order of $10 \mathrm{eV}$. A force of 0.27 Newtons can be measured with this instrument before the linearity of the strain gauges is compromised.

While this instrument was built for and tested in magnetized plasmas, the concept also works for non-magnetized plasmas. The target size or the plasma wetted area may be varied to measure momentum flux at different radial positions of the beam giving a radial profile. Given certain assumptions, the momentum flux data may also be used to infer thrust from the exhaust of a plasma thruster such as the Variable Specific Impulse Magnetoplasma Rocket (VASIMR).'

\section{Concept and Construction}

The momentum flux measuring instrument uses uniaxial strain gauges to sense the force on a target plate. Although strain gauges have been used in pressure gauges and force sensors for many years, the application of semi-conductor strain gauges to sense pressure or momentum flux 
of a plasma flow is unique. Another instrument using a capacitance manometer has been developed that measures plasma momentum flux but the construction is notably different from the instrument described in this article ${ }^{2}$. The strain gauge approach allows for easier integration into the existing test facility and allows for more versatile use of the instrument.

The target plate is immersed in the plasma while the strain gauges must be located outside the plasma flow or must be shielded. The strain gauges in this instrument are placed outside the plasma flow as shown in Fig. 1. An alumina rod is used to electrically and thermally isolate the target plate from the strain gauge component since the target is placed in the plasma flow where it is heated and electrically charged. Besides providing insulation, the alumina rod also serves as a moment arm and increases the strain in the titanium beam for a given force as the rod length is increased.

Strain gauges mounted to the $6 \mathrm{AL}-4 \mathrm{~V}$ titanium beam are used to determine the strain in the titanium. A calibration is required to correlate the strain in the beam to the force of the plasma on the target. The titanium beam also serves as the mounting interface between the force sensor and a rigid mount. Mechanical clamps are used to provide rigid connections between the titanium beam and the alumina rod and also between the target and the alumina rod. These clamps were used instead of epoxy or other adhesive to allow the targets and rods to be changed for different test conditions.

The beam made of 6AL-4V titanium, is schematically shown in Fig. 2 with the strain gauges attached. The design of the beam enhances the stress at the strain gauge location by reducing the area moment of inertia of the cross section. This is accomplished by placing two holes in the beam, one on each side of the two pairs of strain gauges. The stress concentration is 
not uniform over the length of the strain gauges but this effect is accounted for by calibration. The moment of area is given by

$$
I=\frac{b h^{3}}{12}
$$

where $b=2.0 \mathrm{~mm}$ and $h=1.6 \mathrm{~mm}$ as observed in Fig. 2. Using the two holes on each side of the strain gauges to reduce $b$ from $12.7 \mathrm{~mm}$ to $2.0 \mathrm{~mm}$, the moment of area is reduced by $82 \%$. The stress in the titanium beam at the strain gauges location is given by,

$$
\sigma=\frac{M y}{I}
$$

where $M$ is the bending moment, $y$ is the distance from the neutral axis, and $I$ is the moment of area about the neutral axis. The strain, $\varepsilon$, is then related to the stress, $\sigma$, via Hooke's Law which states,

$$
\varepsilon=\frac{\sigma}{E}
$$

where $E$ is Young's tensile modulus and for titanium, $E=1.14 \times 10^{11} \mathrm{~Pa}$. This value of Young's modulus was considered constant for the duration of the tests since the temperature of the beam was measured and determined to remain at ambient temperture.

Assuming a force of nine milli-Newtons ( 0.002 pounds) applied perpendicularly to the target $21.6 \mathrm{~cm}$ from the strain gauges, the bending moment is calculated to be $0.0019 \mathrm{Nm}$. The bending stress is then calculated to be

$$
\sigma=\frac{M y}{I}=\frac{(0.0019) \times(0.00080)}{6.8 \times 10^{-13}}=2.27 \mathrm{MPa}
$$

Equation (2) is used to calculate the strain,

$$
\varepsilon=\frac{2.27 \mathrm{MPa}}{1.14 \times 10^{5} \mathrm{MPa}}=20 \text { micro-strains. }
$$


For a force of one milli-Newton the strain becomes 2.2 micro-strains. A micro-strain is defined as a strain of $10^{-6}$ meters/meter.

One criterion for selecting strain gauges is the micro-strain range expected for the application. The strain gauges tested were semiconductor strain gauges manufactured and mounted by Micron Instruments, Simi Valley, California ${ }^{3}$. The gauge material is Czochralski pulled boron doped silicon. The leads from the strain gauges to the solder pad are $0.05 \mathrm{~mm}$ diameter gold wires which are $12.7 \mathrm{~mm}$ long. The strain gauge is $1.5 \mathrm{~mm}$ long, $0.15 \mathrm{~mm}$ wide, and $0.01 \mathrm{~mm}$ thick. The linearity of these gauges is quoted to be better than $\pm .25 \%$ up to 600 micro-strains. The resistance of the gauges at 26 degrees $C$ is $1,125 \pm 75 \mathrm{Ohms}$ and the gauge factor is $155 \pm 10$. The schematic of the strain gauge placement and numbering are shown in Fig. 3. The four gauges are labelled R1, R2, R3, and R4 and act as resistors in the electrical circuit. ${ }^{4}$ It is the change in resistance of the strain gauge as a function of strain that allows a change in voltage output to be correlated to a force on the target.

Using the Wheatstone bridge configuration as in Fig. 3, the output voltage may be correlated to the input voltage by ${ }^{5}$

$$
V_{\text {out }}=V_{\text {in }}\left(\frac{R_{3}}{R_{3}+R_{4}}-\frac{R_{2}}{R_{1}+R_{2}}\right)
$$

The gauge factor, $G F$, is a measure of the change in resistance of the strain gauge for a given strain where ${ }^{5}$

$$
G F=\frac{\Delta R}{R} \frac{1}{\varepsilon}
$$

Consider the strain caused by the force of nine milliNewtons on the target. The change in resistance of the strain gauge is given by 


$$
\begin{aligned}
& \Delta R=(R)(G F)(\varepsilon) \\
& =(1125)(155)\left(20 \times 10^{-6}\right)=3.5 \Omega .
\end{aligned}
$$

Using Equation (6) and providing an input (excitation) voltage of $10 \mathrm{VDC}$, the change in output (signal) voltage as a result of the applied force is expected to be $31 \mathrm{mV}$. By using the Wheatstone bridge configuration, torsion of the beam about the vertical axis is cancelled by the bridge action. ${ }^{6}$

A frequency of approximately $10.8 \mathrm{~Hz}$ was noticed in the raw data output from the strain gauge. Due to the geometric complexity of the titanium beam holding the strain gauges, the spring constant of the titanium beam was determined experimentally. To determine the spring constant of the system and verify that $10.8 \mathrm{~Hz}$ is the natural frequency of the mechanical system, the strain gauge beam was externally excited without the alumina rod and metal target attached. The force sensor data were then used to determine a torsional spring constant for the titanium beam on which the strain gauges were mounted. As can be seen in Fig. 4 the dominant frequency for the beam after being excited and under no load was measured to be approximately $600 \mathrm{~Hz}$. Using the frequency observed in Fig. 4 the torsional spring constant, k, was calculated using the model outlined in Fig. 5. The alumina rod is assumed rigid based on the stiffness of alumina. Also the instrument is mounted with the alumina rod nearly parallel to the local gravity vector. Using the moment equation about point $\mathrm{O}$,

$$
\sum M_{\theta}=I_{o} \theta^{\prime \prime}
$$

where

$$
\sum M_{\theta}=-k \theta-W \frac{l}{2} \sin (\theta)
$$

The second term in Equation (10) is very small compared to the first and will be neglected. Therefore, 


$$
-k \theta=I_{o} \theta^{\prime \prime}
$$

or

$$
\theta^{\prime \prime}=-\frac{k}{I_{O}} \theta
$$

The solution of this equation has the form,

$$
\theta=A \cos (\omega t+\delta)
$$

where $A$ and $\delta$ are constants determined by initial conditions. The initial conditions may be chosen to allow $A=1$ and $\delta=0$ giving,

$$
\theta^{\prime \prime}=-\omega^{2} \theta
$$

Combining equations (12) and (14) gives

$$
k=\omega^{2} I_{O}
$$

where $\omega=2 \pi f$ and $f$ is the frequency measured in the experiment. The inertia is calculated by assuming the beam from the strain gauges to the end of the beam is rigid and rotates about the axis of the strain gauges, that is point $\mathrm{O}$ in Fig. 5. The mass of this portion of the beam is product of the volume of this portion of the beam and the density of titanium $6 \mathrm{AL}-4 \mathrm{~V}$. The moment of inertia is given by

$$
I_{o}=\frac{1}{12} m\left(a^{2}+4 b^{2}\right)
$$

where $a=.15 \mathrm{~cm}$ and $b=3 \mathrm{~cm}$. This gives

$$
I_{o}=8 \mathrm{gr} \cdot \mathrm{cm}^{2}
$$

Therefore, the torsional spring constant is given by

$$
k=(2 \pi f)^{2} I_{o}=11 N \cdot m
$$

where $f=600 \mathrm{~Hz}$. 
Using the geometry in Fig. 5 to calculate the moment of inertia of the alumina rod about the point $\mathrm{O}$ gives

$$
I_{o}=3,104 \mathrm{gr} \cdot \mathrm{cm}^{2}
$$

Using this value and calculating the expected natural frequency gives

$$
f=\frac{1}{2 \pi} \sqrt{\frac{k}{I_{o}}}=29.9 \mathrm{~Hz} .
$$

As can be seen in Fig. 6, the measured frequency is $28.8 \mathrm{~Hz}$.

The same method was used with the target attached to compare the calculated and measured frequencies. The calculated natural frequency for the sensor with the target attached is 10.3 Hz. As seen in Fig. 7 the measured frequency is $10.8 \mathrm{~Hz}$.

\section{Calibration}

The calibration method used gravity and known masses to apply known forces to the force sensor. The calibration setup is shown in Fig. 8. Output from the force sensor was collected using several different masses. The string was attached at one end to the rear of the target and the other end was fastened to a rigid structure. The string length was varied until the section of string between the calibrated mass and the rigid structure was at an angle of 45 degrees to the horizontal and the section of string between the mass and target was parallel to the horizontal.

The curves plotted using the collected data revealed a linear relationship between the force applied and the signal output of the sensor. A typical calibration curve is shown in Fig. 9. The calibration setup inside the vacuum chamber (not at vacuum) is shown in Fig. 10 with the force sensor in the test position. The discrete data points represented by the data markers are the actual data while the line represents the trend. 


\section{Momentum flux derivation}

This instrument was used in the exhaust of the Variable Specific Impulse Magnetoplasma Rocket (VASIMR) test facility. This facility also has electrostatic probes near the momentummeasuring probe which provide useful plasma conditions such as electron density and temperature as well as the ion flow velocity. Since electrostatic probes only measure plasma properties, there is no method for determining the neutral energies from electrostatic probes. Using the derivation below, the expected momentum flux from the charged particles may be calculated from the electrostatic probe data. The momentum equation along the axial direction (perpendicular to the target plate) for the charged particles is

$$
\frac{d}{d x}\left(m_{i} n_{i} v_{i}^{2}+p_{i}+p_{e}\right)=0
$$

where ionization and collisions are neglected in the exhaust and the electron thermal velocity is much larger than the electron flow velocity. ${ }^{7}$ The electrostatic pressure may be included but the effect averages to zero when the electrostatic pressure on the downstream side of the probe is considered. Also, the electric field can not exert a net force on the plasma since the plasma is quasi-neutral. The electric field at the floating plate tends to reflect electrons and accelerate ions toward the plate with equal and opposite forces. Equation (21) then leads to

$$
m_{i} n_{i} v_{i}^{2}+p_{i}+p_{e}=\text { const }
$$

where $p_{i}=n k T_{i}$ and $p_{e}=n k T_{e}$. To evaluate the constant consider a control volume such that the control volume extends far upstream into plasma. Let this location be at the electrostatic probe axial location where the electron temperature, density, and ion velocity maybe determined and assume $T_{i} \approx 0$. Therefore, $p_{i} \approx 0$ and

$$
n_{i 1} m_{i} v_{i 1}^{2}+n_{e 1} k T_{e 1}=\text { const }
$$


At the floating plate, $p_{e} \approx 0$ since most electrons are reflected due to the sheath electric field, $E$. Therefore,

$$
n_{i p} m_{i} v_{i p}^{2}=n_{e 1} k T_{e 1}+n_{i 1} m_{i} v_{i 1}^{2}
$$

at the plate. The subscript 1 refers to the electrostatic probe axial location and the subscript $p$ refers to the plate axial location. The ion momentum flux to the solid surface defined as

$$
\frac{d P}{d t} \equiv n_{i p} m_{i} v_{i p}^{2}
$$

is obtained from equation (24),

$$
\frac{d P}{d t} \equiv n_{i p} m_{i} v_{i p}^{2}=n_{e 1} k T_{e 1}+n_{i 1} m_{i} v_{i 1}^{2}
$$

Therefore the net pressure (force per unit area) on the plate is

$$
p_{p}=n_{e 1} k T_{e 1}+n_{i 1} m_{i} v_{i 1}^{2}
$$

The radial density profile may be also be determined for the electrostatic probes and integrated across the radius to determine the expected net force on the target plate from the charged particles. The momentum flux, however, from the neutral particles for the conditions of the existing experiment reveal force contributions to be less than $10 \%$ of the momentum flux due to the charged particles.

Momentum and energy flux from sputtered particles were neglected in the above derivation since the threshold energy for sputterning to exist is $21.8 \mathrm{eV}$ for the titanium target and the impact energy of the ions is approximately 15 to $20 \mathrm{eV}$. One effect on the upstream plasma from the titanium target is due to the reflection of electrons toward the upstream direction by the floating potential of the target. Momentum and energy reflection of the impacting particles was determined to be negligable to first order based on the particle and energy reflection coefficients determined by Stangeby. ${ }^{7}$ 
Another concern is the effect of the neutral background density on the plasma between the plasma source and the momentum flux target. The momentum flux target is located axially downstream in a magnetic nozzle region of the existing experiment. The mean free path for charge exchange collisions is changing, getting shorter, throughout the plasma pulse since the neutral background density is increasing during the pulse. The vacuum pumping capacity is inadequate to maintain the low vacuum at the gas flowrates used in the experiment.

\section{Experimental Demonstration}

The plasma used in this experiment was generated using a helicon source that uses radio frequency waves to heat electrons to ionization energies. The force on the target plate as determined from the strain gauge output is shown in Fig. 11. The data has been filtered to suppress the natural frequency of the system. The initial force rise occurring slightly prior to 0.5 seconds is due to the helium gas flow being turned on. The rise in force occurring at 0.75 seconds is due to the plasma being turned on via the helicon antenna operating at $13.56 \mathrm{MHz}$. The plasma shot lasts two seconds. The titanium target immersed in plasma is shown in Fig. 12.

\section{Conclusion}

While strain gauges have been used in similar applications to detect larger forces, application of this technique to detect the small forces from a flowing plasma was not previously available. We have refined a typical strain gauge technique to include these non-trivially small forces and to account for the electrical and thermal noise associated with the plasma generation technique. This instrument has been demonstrated and compared to force values calculated from plasma parameters with good correlation. The details and physics of momentum and heat flux measurements applicable to the plasma thruster will be submitted in a subsequent article. 
${ }^{1}$ F.R. Chang-Diaz, "The Physics and Engineering of the VASIMR Engine", AIAA 20003756 presented at AIAA Joint Propulsion Conference, Huntsville, Alabama, 2000.

${ }^{2}$ S.A. Cohen, F. Zonca, J.Timberlake, T. Bennett, J. Cuthbertson, W. Langer, and R. Motley, Rev. Sci. Instrum. 61, 3586 (1990).

${ }^{3}$ H. Chelner, (private communication, August 2000).

${ }^{4}$ W.D. Cockrell, Industrial Electronics Handbook, (McGraw-Hill, 1958), pp. 6.54-6.56.

${ }^{5}$ F. Kreith, The CRC Handbook of Mechanical Engineering (CRC Press, 1998) p. 5.13; p. 5.8 .

${ }^{6}$ The Pressure, Strain, and Force Handbook, (Omega Press LLC, 2000)

${ }^{7}$ P.C. Stangeby, The Plasma Boundary of Magnetic Fusion Devices, (Institute of Physics Publishing, Bristol and Philadelphia, 2000), p. 46; p. 113. 
FIG. 1. Momentum flux sensor

FIG. 2. Details of titanium beam and strain gauge placement

FIG. 3. a) Schematic of strain gauge location and b) numbering of strain gauges in Wheatstone bridge configuration

FIG. 4. Response of strain gauges with excitation of titanium beam only

FIG. 5. Model of force sensor showing forces with moments and bending axis about point $\mathrm{O}$

FIG. 6. Response of strain gauges with alumina rod attached to titanium beam

FIG. 7. Response of strain gauges with alumin rod and target attached to titanium beam (test configuration)

FIG. 8. Calibration scheme. Mass, $\mathrm{m}$, was varied to generate calibration curve

FIG. 9. Typical calibration curve for force sensor using known forces applied to force target

FIG. 10. In-chamber calibration of force sensor

FIG. 11. Force data for helium plasma impacting $5 \mathrm{~cm}$ diameter target

FIG. 12. Momentum flux titanium target in plasma flow 


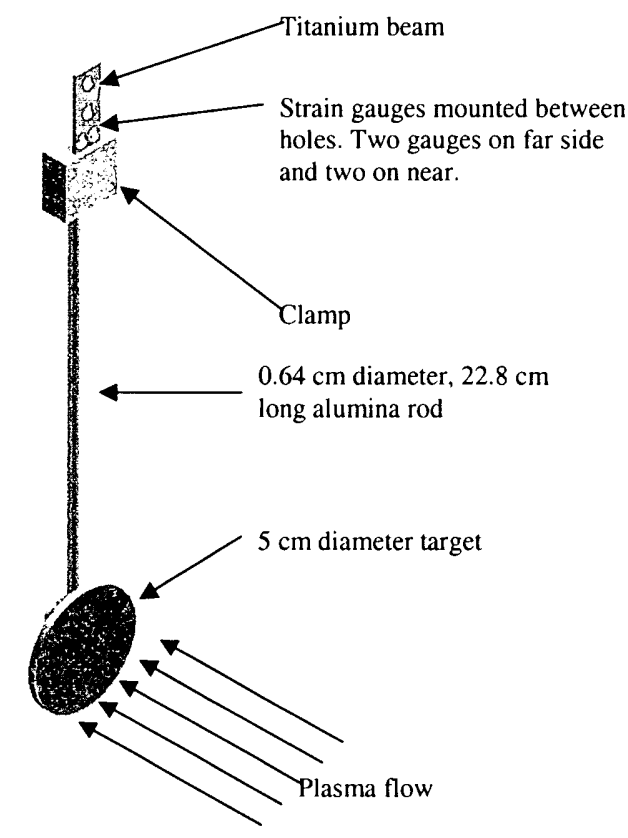

FIG. 1. Momentum flux sensor 


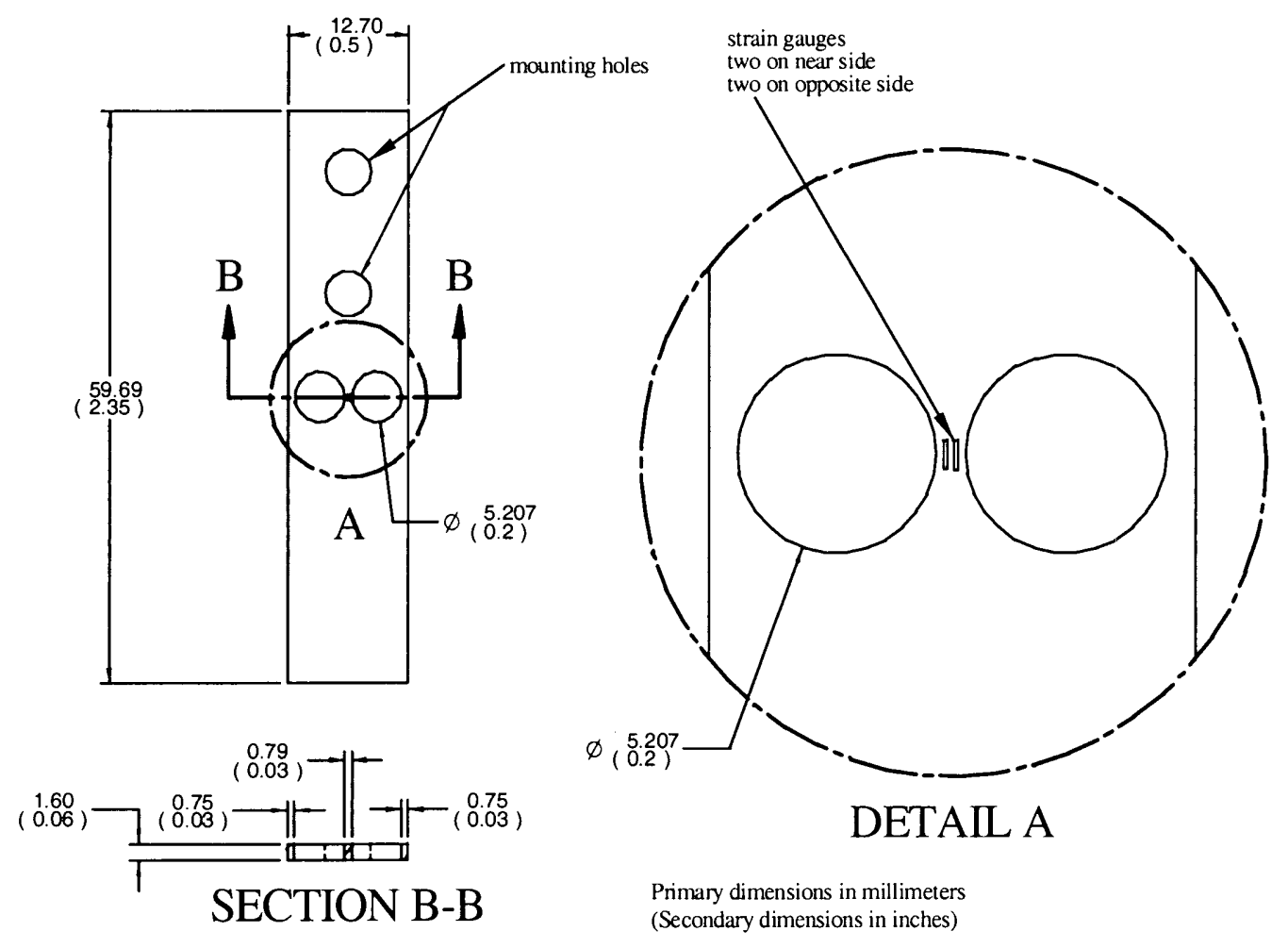

FIG. 2. Details of titanium beam and strain gauge placement. 

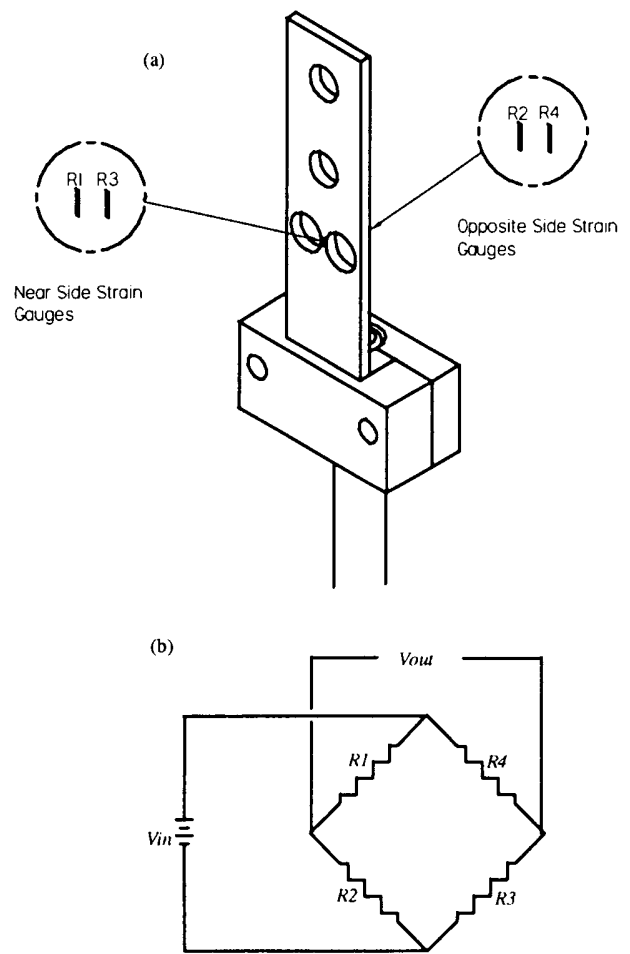

FIG. 3. a) Schematic of strain gauge location and b) numbering of strain gauges in Wheatstone bridge configuration 

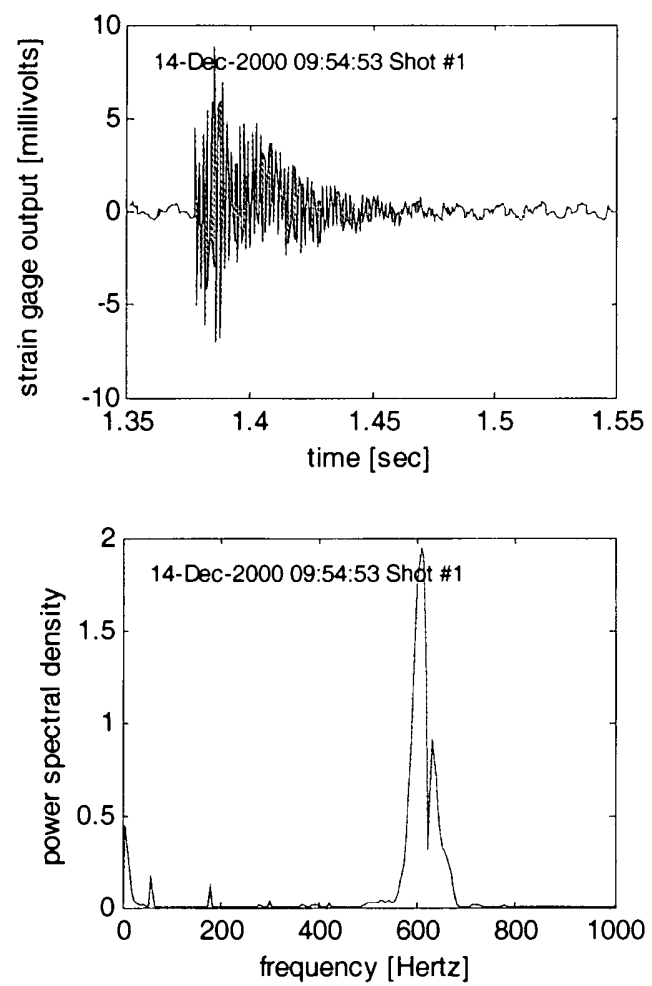

FIG. 4. Response of strain gauges with excitation of titanium beam only 


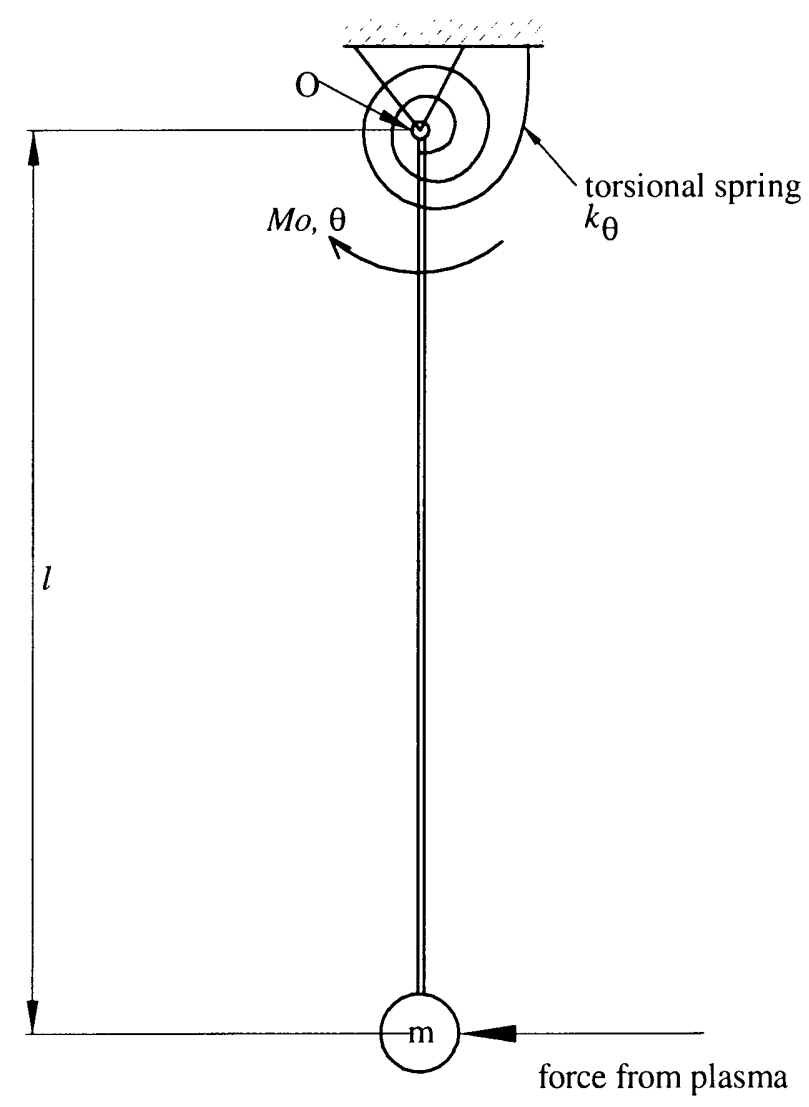

FIG. 5. Model of force sensor showing forces with moments and bending axis about point $\mathbf{O}$. 

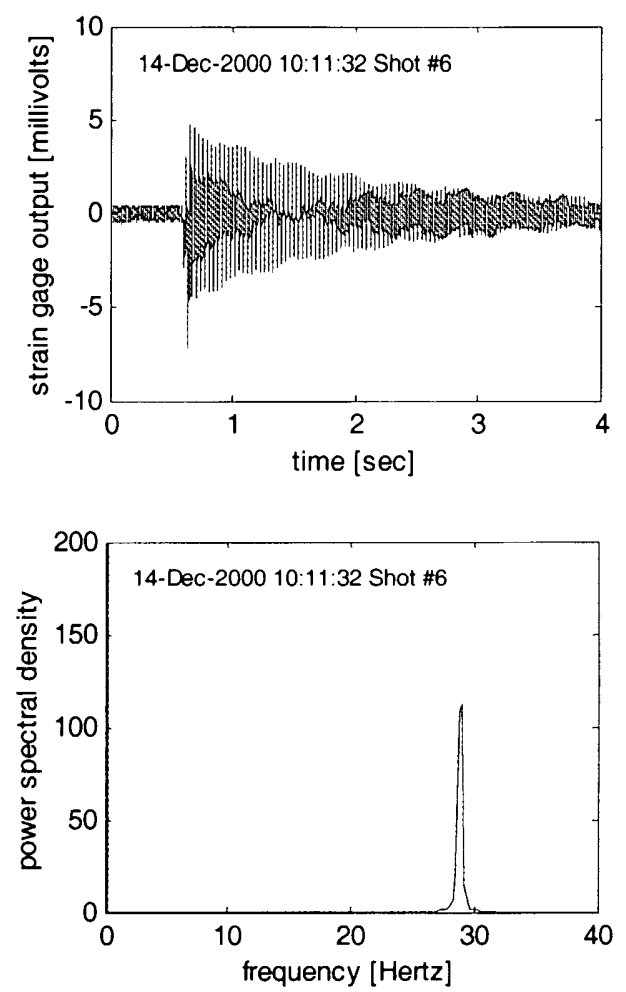

FIG. 6. Response of strain gauges with alumina rod attached to titanium beam 

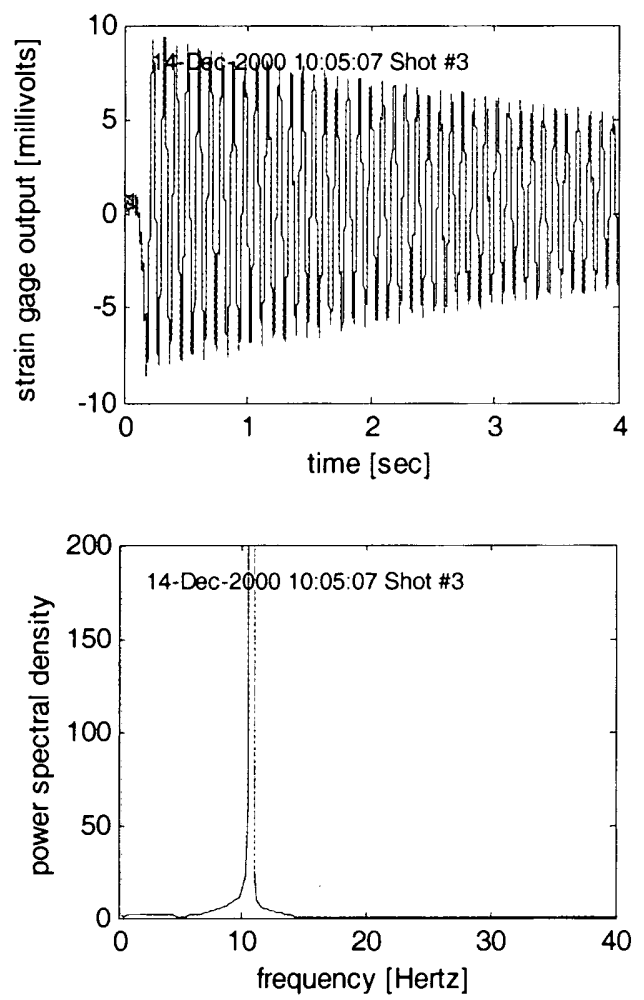

FIG. 7. Response of strain gauges with alumina rod and target attached (test configuration) 


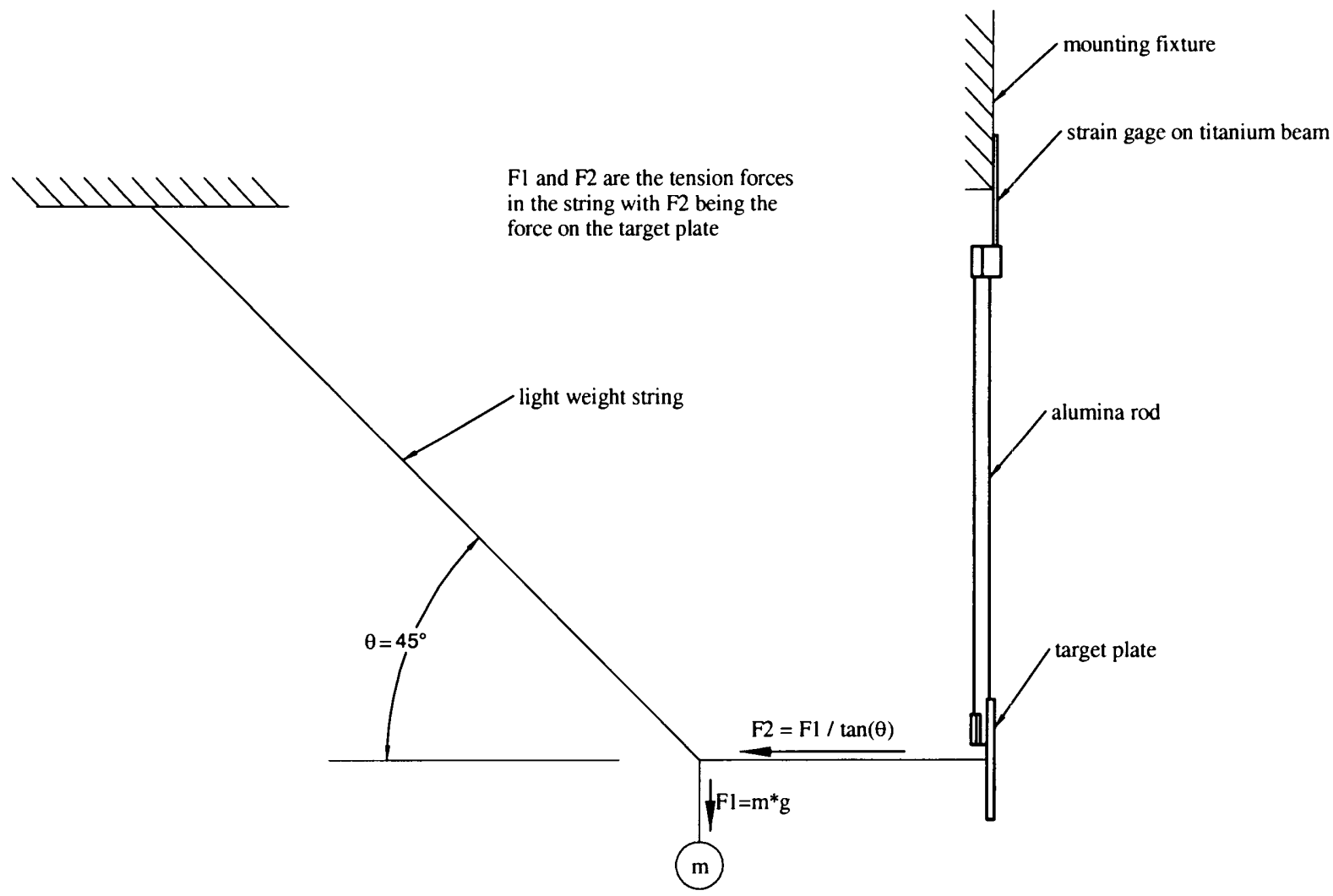

FIG. 8. Calibration scheme. Mass, $\mathrm{m}$, was varied to generate calibration curve 


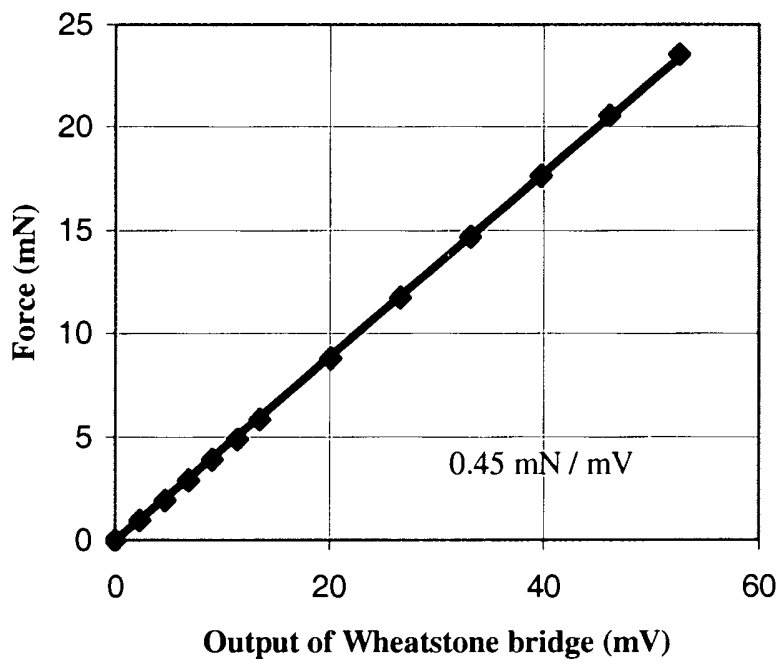

FIG. 9. Typical calibration curve for force sensor using known forces applied to force target 


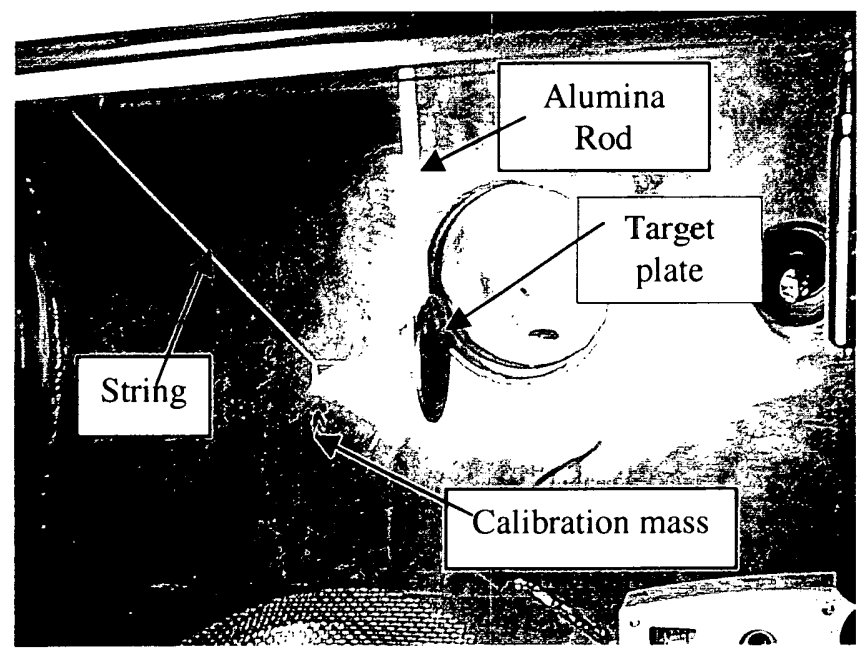

FIG. 10. In-chamber calibration of force sensor 


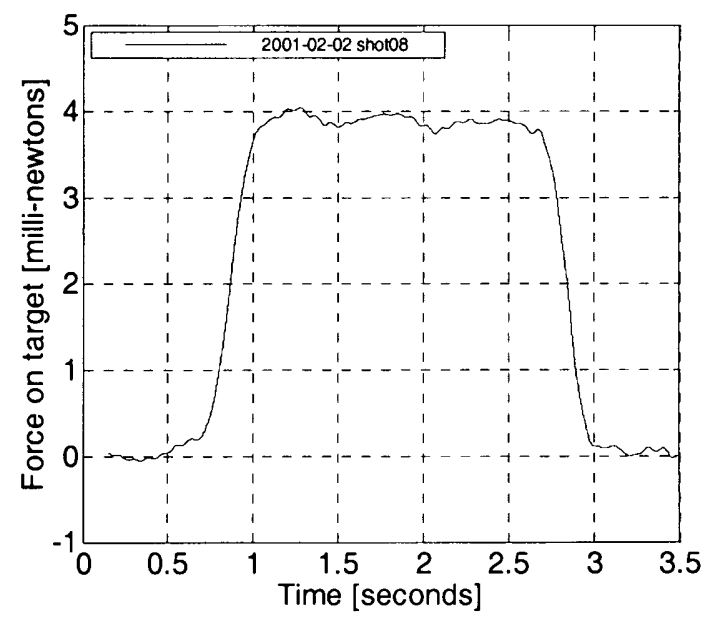

FIG. 11. Force data for helium plasma impacting $5 \mathrm{~cm}$ diameter target 


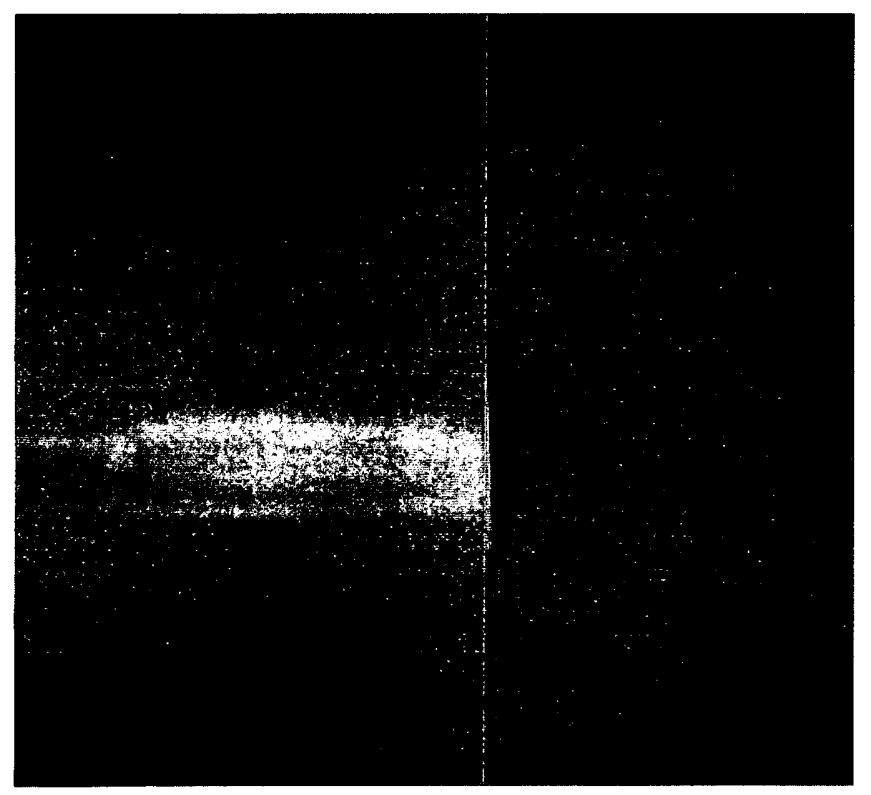

FIG. 12. Momentum flux, titanium target in plasma flow 